\author{
D.A. TULEUOVA', G.A. SERIKBAE ${ }^{1}$, A.K. KURMANALIE ${ }^{1}$, J.U. PYSANOVA, \\ Sh.P. NAJIBULO', E.A. VOSKANYAN ${ }^{1}$ \\ ${ }^{1}$ Kazakh Research Institute of Oncology and Radiology, Almaty, Kazakhstan
}

\title{
Immediate results of electrochemotherapy for malignant tumors of the skin and soft tissues, cutaneous and subcutaneous metastases of melanoma
}

Relevance: Surgical removal of locally advanced forms of skin cancer and multiple cutaneous and subcutaneous metastases of melanoma does not always provide satisfactory aesthetic, functional, and long-term results. The treatment outcomes depend on the size, shape, location, and some other characteristics of the tumor. The relapse rate after surgical removal of locally advanced skin cancer is $12.5-34 \%$, and the long-term survival of patients with cutaneous and subcutaneous metastases of melanoma does not exceed $30 \%$. Cutaneous and subcutaneous metastases of melanoma and some superficial tumors are usually initially inoperable. Their complications such as bleeding, infection, pain, and pitting cause serious clinical problems and often require local therapy even at the 4th stage of the disease.

Electrochemotherapy (ECT) is one of the modern treatment methods for skin cancer, soft tissue sarcoma, and metastatic melanoma. ECT is a treatment of choice in the presence of contraindications to surgical and radiation therapy. ECT combines the physical properties of current-induced electroporation with the chemical properties of chemotherapeutic drugs. In this method, an electric current affecting the tissue causes a temporary increase in cell membrane permeability, thereby providing free access to the cell of large molecules, including cytostatic drugs, which are not initially transferred to the cytosol. This significantly increases the potential toxicity of cytostatic drugs.

The purpose of this study was to demonstrate the immediate results of ECT in patients with malignant tumors of the skin, soft tissue, cutaneous, and subcutaneous melanoma metastases.

Results: Since 2017, 27 patients were treated by ECT at the Centre for bone and soft tissue tumors and melanomas of Kazakh Institute of Oncology and Radiology (Almaty, Kazakhstan). After two months, 67\% of patients had a complete response (CR), 26\% - a partial response (PR), and 7\% had progression of the tumor. Side effects included local edema $(n=13,40 \%)$ which independently disappeared within several days to 2 weeks, local pains (12\%) which disappeared within six months in $3 \%$ and within a month after treatment in $11 \%$, and hyperthermia in the early postoperative period in $6 \%$ of patients which was stopped within 10-15 min after the procedure.

Keywords: electrochemotherapy (ECT), skin cancer, soft tissue sarcoma, cutaneous and subcutaneous metastases, treatment outcome.

Conduct: Electrochemotherapy (ECT) is a combination treatment that utilizes the administration of chemotherapeutic drugs in association with cell membrane electroporation (EP). The method is based on the local application of short, intense electrical impulses which make tissue cells permeable for a short time. To date, ECT is mainly used to treat tumor neoplasms with electrical impulses in the case when non-penetrating drugs acquire high internal cytotoxicity. Improved drug delivery can significantly increase the effectiveness of a chemotherapeutic drug locally at the site of current-induced permeabilization without damaging tissues not exposed to electrical pulses [1].

Electroporation is a physical phenomenon of increasing the cell membrane permeability for drugs due to the use of short and intense electrical impulses. The corresponding number, amplitude, and length of the pulses provide a physically reversible increase of cell membrane permeability.

The first clinical study of ECT with Bleomycin conducted in 1991 by Mir et al. at the Gustave Roussy Institute in France demonstrated good antitumor efficacy in cutaneous metas- tases of the head and neck in patients with carcinoma. In future clinical studies on ECT Bleomycin was administered onsite or IV. Preclinical and clinical studies prove that electric impulses increase the cytotoxicity of Bleomycin up to 1000 times, and Cisplatinum - by 80 times [2].

In 2006, the multicenter project on the elaboration of European Standard for Electrochemotherapy Operating Procedures (ESOPE) approved and published standard operating procedures for the safe and effective use of ECT in clinical practice. In March 2013, NICE issued specific guidance on the use of ECT with metastasis of melanoma skin cancer and non-melanoma skin cancer to the skin and subcutaneous tissue. Over the past 20 years, ECT has demonstrated proven efficacy in the treatment of tumors of the skin, subcutaneous, mucous membrane, or deep tumors, with different histological structure and in different parts of the body. ECT is also effective for bleeding tumors [ 3 ].

The purpose of this study was to demonstrate the direct outcome of ECT treatment of patients with malignant tumors of the skin and soft tissues, skin and subcutaneous metastases of melanoma. 
Materials and Methods: The Kazakh Institute of Oncology and Radiology (KazIOR) applies ECT with Bleomycin (or Cisplatinum) since January 2017. All patients were treated at the Center for Tumors of Bones, Soft Tissues and Melanoma of KazlOR.

All tumors were localized on the skin or within soft tissues to a depth of $3.0 \mathrm{~cm}$ from the skin surface.

Indications for ECT included: progressive primary or recurrent skin neoplasm (skin cancer); bleeding, painful, and decaying tumors; skin and subcutaneous metastases of neoplastic lesions, regardless of their histology, located within $3 \mathrm{~cm}$ from the skin surface; the elderly age of patients with an increased risk of side effects and toxicity associated with other methods due to concomitant diseases; Kaposi's sarcoma; soft tissue sarcoma; vulvar cancer.

Contraindications included: clinical manifestations of arrhythmia or an installed pacemaker; pulmonary fibrosis; epilepsy; active infection; known allergy to Bleomycin; renal failure; previous treatment with Bleomycin in the maximum cumulative dose.

Treatment: According to the ESOPE protocol, ECT was performed under general or spinal anesthesia after preliminary intravenous administration of Bleomycin at a dose of $15,000 \mathrm{lU} / \mathrm{m} 2$ body surface area (or Cisplatinum at a dose of $20 \mathrm{mg} / \mathrm{m} 2$ body surface area). Electric currents were delivered with a needle electrode 1-3 cm long. The electrodes were connected to an impulse generator. High voltage up to $1000 \mathrm{~V}$ was delivered as a compressed circuit of eight pulses with the frequency of $5000 \mathrm{~Hz}$ for $100 \mu \mathrm{s}$. The pulses were delivered in the interval of 8 to 28 minutes after intravenous administration of the drug [4]. The procedure was scheduled and inpatient. The average hospital stay of a patient was seven days.

The tumor volume was calculated. The response to treatment was classified in accordance with WHO guidelines: complete response (CR) - the tumor is not palpable; partial response (PR) - volume reduction by more than $50 \%$; no change - an increase in the volume by less than $25 \%$ or a decrease by less than $50 \%$; disease progression an increase in the volume by more than $25 \%$.

All responses were evaluated six weeks after the first ECT procedure.

Patients were observed once a week for the first six weeks, and then once a month. At each examination, tumors were measured and photographed.

Results: From January 2017 to March 2020, ECT was performed 32 times in total in 27 patients: 27 times with Bleomycin and 5 with Cisplatinum (Table 1). The average number of electrode applications - 84 per session. Only hexagonal electrodes were used.

Table 1 - Distribution of cases by year, histological type of tumor

\begin{tabular}{|c|c|c|c|c|c|c|}
\hline $\begin{array}{l}\text { Histological type of } \\
\text { tumor }\end{array}$ & No. of pro-cedures & 2017 & 2018 & 2019 & 2020 & Chemotherapy drug \\
\hline Basal cell carcinoma & 6 & 1 & 1 & 2 & 2 & $\begin{array}{c}\text { Bl-5 } \\
\text { Cisp-1 }\end{array}$ \\
\hline $\begin{array}{l}\text { Squamous cell carci- } \\
\text { noma }\end{array}$ & 4 & 2 & & 2 & & $\mathrm{Bl}-4$ \\
\hline Sarcoma Kaposi & 5 & 3 & 1 & & 1 & $\begin{array}{c}\text { Cisp-2 } \\
\text { Bl-3 }\end{array}$ \\
\hline $\begin{array}{l}\text { Subcutaneous MTS } \\
\text { Melanoma }\end{array}$ & 10 & 4 & 3 & 3 & & $\begin{array}{l}\text { Cisp-2 } \\
\text { Bl-8 }\end{array}$ \\
\hline Soft tissue sarcoma & 3 & & 1 & 2 & & $\mathrm{Bl}-3$ \\
\hline Vulvar cancer & 3 & & 1 & & 2 & $\mathrm{Bl}-3$ \\
\hline Lower lip cancer & 1 & & 1 & & & $\mathrm{Bl}-1$ \\
\hline Total & 32 & 10 & 8 & 9 & 5 & \\
\hline
\end{tabular}

Of the 27 patients who underwent ECT, 22 (81\%) patients received one course, and $5(19 \%)$ - two courses. 12 (44\%) patients were male, $15(56 \%)$ - female. Most of the patients were 60 to 70 years old - $16(59 \%)$, over 70 years old - 7 (26\%), 45-59 years old - 4 (15\%).

Adverse Reactions. An adverse reaction in the form of local edema was observed in 13 (40\%) patients and independently disappeared within a few days to 2 weeks. Local pains were observed in $4(12 \%)$ patients, of which one had local pains for six months, and three patients persisted for a month after treatment. The phenomena of hyperthermia in the early postoperative period were observed in $2(6 \%)$ and were stopped within 10-15 minutes after the procedure. No other serious adverse reactions and hematological toxicity to Bleomycin and Cisplatinum were observed (Table 2).
Table 2 - Side effects after ECT

\begin{tabular}{|l|c|c|}
\hline \multicolumn{1}{|c|}{ Side effects } & No. of patients & Duration \\
\hline Local edema & $13(40 \%)$ & $5-14$ days \\
\hline Local pain & $4(12 \%)$ & $1-6$ months \\
\hline $\begin{array}{l}\text { Hyperthermia in the } \\
\text { early postoperative } \\
\text { period }\end{array}$ & $2(6 \%)$ & $10-15$ minutes \\
\hline
\end{tabular}

Of the 27 treated patients, 6 (22\%) had basal cell carcinoma (stage II - 3 patients, stage III - 1), squamous skin cancer3 (11\%) patients with stage III, Kaposi's sarcoma - 4 (14\%), subcutaneous MTS of melanoma - $8(30 \%)$, soft tissue sarcoma - $2(7 \%)$ patients (soft-tissue fibroma of the lower limb, the condition after complex treatment, a relapse), vulvar cancer - 3 (11\%), lower lip cancer stage II - 1 (4\%). 
The patients who underwent two courses of ECT included: 5 (18\%) patients with skin cancer recurrence and a partial response in subcutaneous metastases of melanoma and 1 (4\%) patient with the continued growth of soft tissue tumor on the lower extremity.

A progression was observed in 2 (7\%) patients with soft tissue dermatofibroma of the lower limb; the continued tumor growth was observed one month after treatment in 3 (11\%) patients with subcutaneous metastases of melanoma.

Two months after ECT, 18 (67\%) out of 27 patients have achieved a complete response, 7 (26\%) - a partial response, and 2 (7\%) - progression. The follow-up period for ten patients treated in 2017 was at least two years. Six $(60 \%)$ have achieved a local response, three $(30 \%)$ showed repeated manifestations at the ECT site after five months and one year. In eight patients treated in 2018, the follow-up period was one year. Local response was achieved in $4(50 \%)$ patients.

\section{Discussion:}

In this article, we report the results of treatment of 27 patients with primary or recurrent skin and subcutaneous malignant tumors using ECT in combination with intravenous Bleomycin (or Cisplatinum). ECT was performed using a Cliniporator apparatus in accordance with ESOPE standard operating procedures. This method is based on increasing the cell membrane permeability due to the creation of transition pores under the influence of electric fields. The increased permeability promotes direct diffusion of various molecules into malignant cells [4].

Currently, only Bleomycin and Cisplatinum are used in combination with ECT. When administered separately, Bleomycin is usually bound, and less than $0.1 \%$ of it crosses the cell membrane. However, in combination with ECT, the intracellular concentration of Bleomycin increases by 700-1000 times what in turn increases its cytotoxicity by several thousand times. The intracellular concentration of Cisplatin In combination with electrical impulses increases by only 80-100 times.

ECT is an effective and safe treatment for the local control of inoperable skin and subcutaneous lesions with a surgery rate of 80 to $90 \%$. Using ECT as neoadjuvant and independent treatment allows avoiding destructive surgery while achieving oncological radicalism and ensuring the aesthetic and functional ability of the organ. In the treatment of bleeding and painful tumors, ECT also effectively relieves symptoms and significantly improves the quality of life of patients.

\section{Conclusion:}

The achieved treatment outcomes prove ECT to be a simple, new, fast, and easily manageable treatment in terms of local and systemic toxicity, cost-effectiveness ratios, and good cosmetic results. We would also like to emphasize the role of ECT as the final and palliative treatment of skin and subcutaneous tumors, regardless of their histology and previous treatment. The method is well tolerated; it is the method of choice for inoperable tumors and tumors resistant to chemoradiotherapy.

\section{References:}

1. Tuleuova D.A., Serikbayev G.A., Pyssanova Zh.U., A.K. Kurmanaliyev, Ormanov K.M. Clinical case: treatment of a patient with an inoperable form of primary-multiple skin cancer by electrochemotherapy // Oncology and Radiology of Kazakhstan. 2017. - №4(46). - P. 38-40;

2. Kreuter A., van Eijk T., Lehmann P. et al. Electrochemotherapy in advanced skin tumors and cutaneous metastases - a retrospective multicenter analysis // J. Dtsch. Dermatol. Ges. - 2015. - Vol. 13. - P. 308-315;

3. Mir-Bonafe J.M., Vilalta A., Alarcon I. et al. Electrochemotherapy in the Treatment of Melanoma Skin Metastases: a report on 31 cases // Actas Dermo-Sifiliogr. - 2015. - Vol. 106. - P. 285-291;

4. Plaschke C.C., Bertino G., McCaul J.A. et al. European Research on Electrochemotherapy in Head and Neck Cancer (EURECA) project: Results from the treatment of mucosal cancers // Eur. J. Cancer. 2017. - Vol. 87. - P. 172-181. 